\title{
Molecular characteristics of the KCNJ5 mutated aldosterone-producing adenomas
}

\author{
Masanori Murakami', Takanobu Yoshimoto', Kazuhiko Nakabayashi2, Yujiro Nakano', \\ Takahiro Fukaishi', Kyoichiro Tsuchiya1, Isao Minami1, Ryotaro Bouchi', \\ Kohji Okamura3, Yasuhisa Fujii4, Koshi Hashimoto1,5, Ken-ichiro Hata², \\ Kazunori Kihara ${ }^{4}$ and Yoshihiro Ogawa1,6,7
}

1Department of Molecular Endocrinology and Metabolism, Graduate School of Medical and Dental Sciences, Tokyo Medical and Dental University, Tokyo, Japan

2Department of Maternal-Fetal Biology, National Research Institute for Child Health and Development, Tokyo, Japan 3Department of Systems BioMedicine, National Research Institute for Child Health and Development, Tokyo, Japan ${ }^{4}$ Department of Urology, Graduate School of Medical and Dental Sciences, Tokyo Medical and Dental University, Tokyo, Japan

5Department of Preemptive Medicine and Metabolism, Graduate School of Medical and Dental Sciences, Tokyo Medical and Dental University, Tokyo, Japan

${ }^{6}$ Department of Medical and Bioregulatory Science, Graduate School of Medical Sciences, Kyushu University, Fukuoka, Japan

7Japan Science and Technology Agency, CREST, AMED, Tokyo, Japan

Correspondence should be addressed to T Yoshimoto Email tyoshimoto.mem@tmd.ac.jp

\begin{abstract}
The pathophysiology of aldosterone-producing adenomas (APAs) has been investigated via genetic approaches and the pathogenic significance of a series of somatic mutations, including KCNJ5, has been uncovered. However, how the mutational status of an APA is associated with its molecular characteristics, including its transcriptome and methylome, has not been fully understood. This study was undertaken to explore the molecular characteristics of APAs, specifically focusing on APAs with KCNJ5 mutations as opposed to those without KCNJ5 mutations, by comparing their transcriptome and methylome status. Cortisol-producing adenomas (CPAs) were used as reference. We conducted transcriptome and methylome analyses of 29 APAs with KCNJ5 mutations, 8 APAs without KCNJ5 mutations and 5 CPAs. Genome-wide gene expression and CpG methylation profiles were obtained from RNA and DNA samples extracted from these 42 adrenal tumors. Cluster analysis of the transcriptome and methylome revealed molecular heterogeneity in APAs depending on their mutational status. DNA hypomethylation and gene expression changes in Wnt signaling and inflammatory response pathways were characteristic of APAs with KCNJ5 mutations. Comparisons between transcriptome data from our APAs and that from normal adrenal cortex obtained from the Gene Expression Omnibus suggested similarities between APAs with KCNJ5 mutations and zona glomerulosa. The present study, which is based on transcriptome and methylome analyses, indicates the molecular heterogeneity of APAs depends on their mutational status. Here, we report the unique characteristics of APAs with KCNJ5 mutations.
\end{abstract}

\section{Key Words}

- molecular genetics

- adrenal cortex

- adenoma

- gene expression 


\section{Introduction}

Aldosterone-producing adenoma (APA) is a subtype of primary aldosteronism (PA), which is the most common form of endocrine hypertension (Plouin et al. 2004, Hannemann \& Wallaschofski 2012) and is more closely associated with severe cardiovascular complications compared to essential hypertension (Milliez et al. 2005). The subtype classification of PA is important from a therapeutic viewpoint because APAs are curable by unilateral adrenalectomy.

Recently, the molecularcharacteristics of APA have been widely investigated via high-throughput technologies, including exome sequencing, transcriptomics (Zennaro et al. 2012) and methylomics (Howard et al. 2014, Murakami et al. 2015b). In particular, exome sequencing studies have uncovered that a series of somatic mutations in genes such as genes encoding the potassium inwardly rectifying channel, subfamily J, member 5 (KCNJ5), ATPase, $\mathrm{Na}^{+} / \mathrm{K}^{+}$transporting, alpha1-polypeptide (ATP1A1), ATPase, $\mathrm{Ca}^{2+-}$ transporting, plasma membrane 3 (ATP2B3), calcium channel, voltage-dependent, L-type, alpha1D-subunit (CACNA1D), calcium channel, voltagedependent, $\mathrm{T}$ type, alpha $1 \mathrm{H}$ subunit $(C A C N A 1 H)$, and catenin beta1 (CTNNB1), lead to autonomous aldosterone production (Choi et al. 2011, Azizan et al. 2013, Beuschlein et al. 2013, Scholl et al. 2015b, Akerstrom et al. 2016). Previous studies of over 1000 sporadic APAs have revealed that KCNJ5 mutations are the most frequent mutations (Lenzini et al. 2015). The prevalence of KCNJ5 mutations in APAs reaches approximately $24 \sim 41 \%$ in Western countries (Akerstrom et al. 2012, Azizan et al. 2012b, Boulkroun et al. 2012, Osswald et al. 2013, Rossi et al. 2014), on the other hand, that in Asian countries reaches 65 82\% (Taguchi et al. 2012, Kitamoto et al. 2015, Wang et al. 2015, Murakami et al. 2016). It has been shown that patients with APAs with KCNJ5 mutations are predominantly female in Western countries (Boulkroun et al. 2012, Fernandes-Rosa et al. 2014, Williams et al. 2014, 2016, Akerstrom et al. 2015, Scholl et al. 2015a) and younger (Boulkroun et al. 2012, Taguchi et al. 2012, Fernandes-Rosa et al. 2014, Akerstrom et al. 2015, Kitamoto et al. 2015, Wang et al. $2015)$ than those without KCNJ5 mutations. In addition, a higher proportion of patients with KCNJ5 mutations have resolved hypertension after adrenalectomy compared to those without KCNJ5 mutations (Arnesen et al. 2013).

In addition to these reports regarding the clinical aspects of APAs with KCNJ5 mutations, gene expression patterns specific to this subtype of APA have been reported in several studies (Akerstrom et al. 2015, Murakami et al. 2016). For example, we have recently shown that inflammation-related genes are downregulated in APAs with KCNJ5 mutations (Murakami et al. 2016). However, detailed transcriptomic distinctions between APAs with KCNJ5 mutations and those with other mutational status or other types of adrenal tumors, such as cortisol-producing adenomas (CPAs), have not as yet been elucidated.

To further investigate APAs at the molecular level, research on acquired epigenetic changes, such as DNA methylation at $\mathrm{CpG}$ dinucleotides, is considered to be important. DNA methylation status is relevant to a variety of physiologic and pathophysiologic conditions, such as cell differentiation and tumorigenesis (Jaenisch \& Bird 2003, Das \& Singal 2004). Indeed, our recent study indicates global hypomethylation in APAs when compared to the adjacent adrenal gland (Murakami et al. 2015b). Aberrant DNA methylation status observed in APAs may contribute to their pathogenesis via modification of gene expression. As our previous study included only six APAs with KCNJ5 mutations and one APA with an ATP1A1 mutation, we were unable to obtain detailed information regarding mutational status in that study.

The present study was undertaken to explore the molecular characteristics of APAs, with a special focus on APAs with KCNJ5 mutations as opposed to those without KCNJ5 mutations, by comparing their transcriptome and methylome statuses in order to gain further insights into the pathogenesis of APAs.

\section{Materials and methods}

\section{Subjects}

The study protocol was approved by the Ethics Committee for Human Research at the Tokyo Medical and Dental University (nos. 191 and 1371). A written informed consent was obtained from all participants.

Adrenal tissue was obtained from 37 patients with APA and five patients with CPA who underwent adrenalectomy at the Tokyo Medical and Dental University Hospital. These 37 patients satisfied the diagnostic criteria for PA based on the Japan Endocrine Society guidelines (Nishikawa et al. 2011), as previously described (Murakami et al. 2015b). The diagnosis of CPA was based on the diagnostic criteria of autonomous cortisol secretion by 'European Society of Endocrinology Clinical Practice Guideline in collaboration with the European Network for the Study

Published by Bioscientifica Ltd 
of Adrenal Tumors' (Fassnacht et al. 2016) as follow: serum cortisol $>50 \mathrm{nmol} / \mathrm{L}(>1.8 \mu \mathrm{g} / \mathrm{dL})$ after 1,2 or $8 \mathrm{mg}$ overnight dexamethasone suppression tests, or 2-day low-dose dexamethasone test, and one of the following additional endocrine alterations: increased 24-h urinaryfree cortisol, low plasma ACTH, elevated midnight serum or salivary cortisol. No overt symptoms of Cushing's syndrome were confirmed by physical examination for all patients. Tumor tissue was macroscopically dissected and stored at $-80^{\circ} \mathrm{C}$. All APAs and CPAs in the present study were from cases that were used in previous studies (Murakami et al. 2015a,b, 2016).

\section{Extraction of genomic DNA and total RNA}

Genomic DNA and total RNA samples were isolated from APA and CPA tissue from the 42 patients using the All Prep DNA/RNA Mini kit (Qiagen, Valencia, CA) according to the manufacturer's instructions.

\section{Detection of somatic mutations in APAs}

APA-specific KCNJ5, ATP1A1, ATP2B3 and CACNA1D mutations were analyzed by PCR and direct sequencing. Detailed methods, including primer information, have been described previously (Murakami et al. 2016).

\section{Next-generation sequencing}

Exome sequencing, RNA-seq analysis and DNA methylation profiling were performed. For further details, see the Supplementary Materials and methods (see the section on supplementary data given at the end of this article).

\section{Gene ontology and pathway analysis for differentially expressed and methylated genes}

The Database for Annotation, Visualization and Integrated Discovery was used to determine enriched Gene Ontology (GO) terms within the selected gene groups (Dennis et al. 2003). We performed biological pathway analysis using the GenMAPP/MAPPFinder software (http://www. genmapp.org). Two-hundred and six annotated human pathways downloaded from the GenMAPP/Wikipathway were used for the analysis. Gene expression data from RNA-seq analysis were mapped to the human pathways. MAPPFinder 2.0 was used to find significant pathways wherein differentially expressed genes were enriched. Permutation test $P$ values were calculated in MAPPFinder to estimate the statistical significance of the enrichment.

\section{Integration of methylome and RNA-seq data}

The values, which are an estimate of methylation level using ratio of intensities between methylated and unmethylated alleles by Illumina BeadChip experiments, were averaged for each category for the same gene. Categories were based on Illumina $450 \mathrm{~K}$ beadchip annotation: TSS: proximal promoter defined as $200 \mathrm{bp}$ (TSS200) or $1500 \mathrm{bp}$ (TSS1500) upstream of the transcription start site, UTR: untranslated region, and ExonBnd: exon boundaries. The CpG sites containing null values for any sample were removed from the analysis. The $\mathrm{CpG}$ sites of chromosome $\mathrm{X}$ and chromosome $\mathrm{Y}$ were also removed from the analysis. The RNA-seq-normalized expression values and averaged values were combined based on the gene symbol. Dimension-reduction analysis for integrated data was performed using the Multiple co-inertia analysis (MCIA) method implemented in the omicade4 (Meng et al. 2014) in $\mathrm{R}$ package.

\section{Comparison of transcriptome data to Gene Expression Omnibus data}

Transcriptome data from 42 adrenal tumors were compared to microarray data from normal adrenal cortex (Gene Expression Omnibus [GEO] accession no. GSE68889), which included transcriptomes for adrenocortical layers, the zona glomerulosa (ZG) and zona fasciculate (ZF), from adrenal glands obtained from four kidney transplantation donors (Nishimoto et al. 2015). The gene expression patterns of $Z G$ relative to $Z F$ were compared using these GEO data (ZG vs ZF). The 42 adrenal tumors were classified into the following four groups based on similarities in transcriptomic profiles: APAs with KCNJ5 mutations (hereinafter abbreviated as 'KA'), APAs with CTNNB1 mutations and without any other known mutations (abbreviated as 'NC'), APAs with ATP1A1, ATP2B3 and CACNA1D mutations (abbreviated as ' $\mathrm{AC}$ ') and $\mathrm{CPA}$. We then compared gene expression patterns between pairs of groups selected from the above four groups (12 paired comparisons: KA vs NC, NC vs $\mathrm{KA}, \mathrm{KA}$ vs AC, AC vs KA, KA vs CPA, CPA vs KA, NC vs CPA, CPA vs NC, AC vs NC, NC vs AC, AC vs CPA and CPA vs AC). Similarities in gene expression patterns between the ZG and ZF and those found in the 12 paired comparisons were evaluated using the method described in Supplementary Fig. 1 (see section on supplementary data given at the end of this article).

Published by Bioscientifica Ltd 


\section{Data deposition}

The data used for this publication have been deposited in the National Center for Biotechnology Information Gene Expression Omnibus and are accessible via the Gene Expression Omnibus. Series accession number GSE90867 was allocated to our data.

\section{Statistical analysis}

Fold changes in RNA-seq data used to compare APAs and CPAs were analyzed using a moderate $t$-test with asymptotic and Benjamini and Hochberg False discovery rate correction. values comparing APAs and CPAs were analyzed using the Wilcoxon rank sum tests with Benjamini and Hochberg False discovery rate correction.

\section{Results}

\section{Patient information}

Clinical characteristics of the 37 patients with APA (18 men, age $49.8 \pm 11.0$ years) and five patients with CPA (one man, age $51.6 \pm 7.8$ years) are described in Supplementary Table 1A and B. All of the patients with APA exhibited hypertension, low plasma renin activity and high plasma aldosterone concentration. Resected specimens were histologically diagnosed as adrenocortical adenoma.

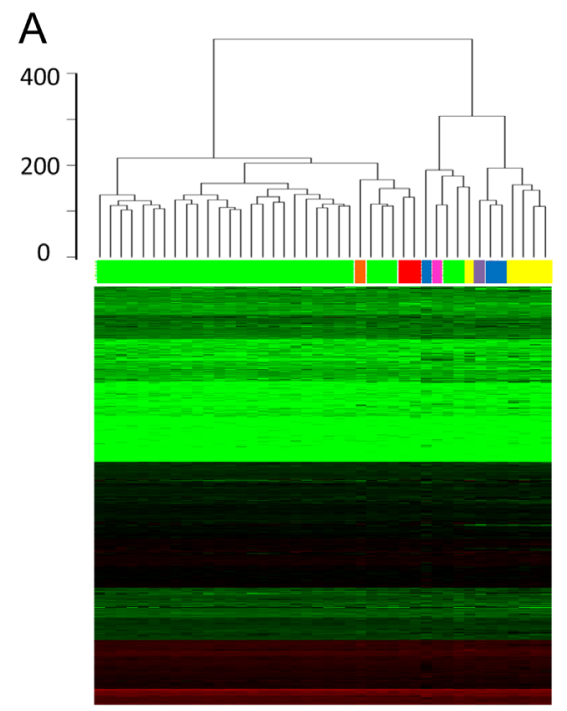

\section{Somatic mutation analysis}

We first performed somatic mutation analysis in our patients (Supplementary Table 2). Sequencing of DNA samples from 29 APAs revealed the presence of heterozygous KCNJ5 mutations. Two adenomas had nucleotide substitutions in ATP1A1, one had a mutation in ATP2B3 and one had a mutation in CACNA1D, as previously described (Murakami et al. 2016). The absence of mutations in all peripheral DNA samples confirmed the somatic nature of the genetic alteration in these patients. As four adenomas did not have the abovementioned mutations, we performed whole exome sequencing to find any other known APA-related mutations, such as CTNNB1 and CACNA1H (Akerstrom et al. 2016, Daniil et al. 2016). We found that one of the adenomas had a nucleotide substitution in CTNNB1. This mutation was reported to be an APA-related mutation in a previous study (Akerstrom et al. 2016). However, no known mutations related to APAs were detected in three adenomas.

\section{Transcriptome analysis}

Global changes in gene expression associated with APA and CPA were evaluated using RNA-seq. Unsupervised hierarchical clustering analysis revealed differential gene expression patterns between APAs and CPAs. APAs had heterogeneous transcriptomic patterns (Fig. 1A).

\section{Figure 1}

Transcriptome and methylome analyses of 37APAs and 5CPAs. (A) Hierarchical clustering and heatmap of the transcriptome profiles based on Euclidean distances in 37 APAs and 5 CPAs. (B) Three-dimensional principal component analysis of the transcriptome profiles in 37 APAs and 5 CPAs. Component ratios used for analysis were PC1: $13.8 \%$, PC2: $9.6 \%$ and $\mathrm{PC} 3: 7.6 \%$.
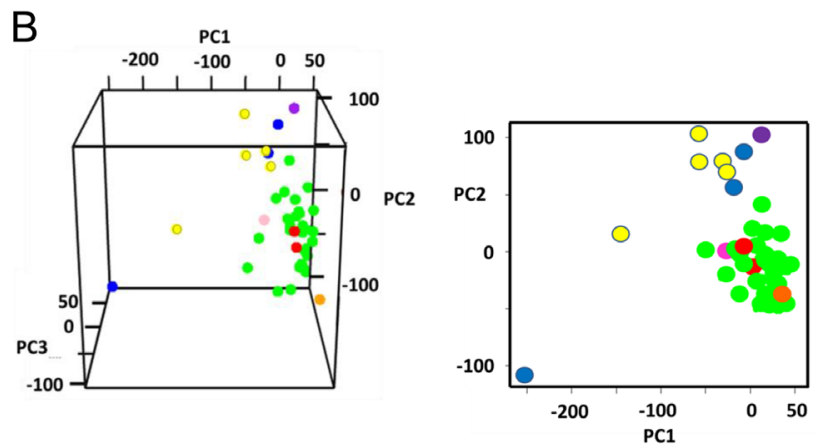
Transcriptomic patterns of APAs with ATP1A1 and CACNA1D mutation were similar to those of APAs with KCNJ5 mutation, otherwise, those of APAs with CTNNB1 and no mutation were closer to those of CPAs than those of APAs with KCNJ5 mutation. Likewise, principal component analysis, which was performed to elucidate the relationships of transcriptome patterns in the point of mutational status, on all probes clearly showed the presence of the same transcriptomic pattern, as shown in the clustering analysis (Fig. 1B). Differentially expressed genes specific to APAs with KCNJ5 mutations, but not APAs without KCNJ5 mutations, were extracted (Supplementary Table 3A and B). We identified 106 genes that were upregulated (more than 2-fold, false discovery rate $[\mathrm{FDR}]<0.05)$ in APAs with KCNJ5 mutations. Sixtyeight genes were downregulated. GO analysis of the differentially expressed genes indicated that GO terms, such as 'plasma membrane' and 'plasma membrane part' in the cellular component (Supplementary Table 3C), 'muscle contraction' and 'muscle system process' in biological process (Supplementary Table 3D), and 'channel activity' and 'passive transmembrane transporter activity' in molecular function (Supplementary Table 3E), were enriched.

\section{Methylome analysis}

The genome-wide CpG methylation patterns of APAs and CPAs were analyzed by calculating the mean value for the entire probe set with unsupervised hierarchical clustering analysis (Fig. 2A). Principal component analysis was also performed to elucidate the relationships of methylome patterns in the point of mutational status (Fig. 2B). Similar to the results of the transcriptome analysis, methylome patterns of APAs were heterogeneous. Scatter plotting of average values indicated invariable CpG hypomethylation status in APAs with KCNJ5 mutations compared to those without KCNJ5 mutations (Fig. 2D). We found 1413 significantly hypomethylated genes ( value $<-0.1$, $P<0.001)$ in APAs with KCNJ5 mutations. However, we only found 13 hypermethylated genes (value $>0.1, P<0.001$ ). The top ten hypomethylated and hypermethylated genes are shown in Supplementary Table 1F and 1G. GO analysis of the hypomethylated genes shows that GO terms, such as 'extracellular region' and 'plasma membrane' in the cellular component (Supplementary Table 3H), 'sensory perception of chemical stimulus' and 'the G-protein coupled receptor protein signaling pathway' in biological process (Supplementary Table 3I), and 'olfactory receptor activity' and 'cytokine activity' in molecular function (Supplementary Table 3J), were enriched.

\section{Integration of the transcriptome and methylome analyses}

To explore distinctions in gene expression and $\mathrm{CpG}$ methylation status between APAs with and without KCNJ5 mutations, we integrated the transcriptome and methylome analyses. At first, we used MCIA as a dimension-reduction technique for data integration (Meng et al. 2014) and confirmed the heterogeneity of APAs and same tendency as observed in each transcriptome and methylome analysis (Fig. 3). We next examined whether CpG methylation status at any functional region (listed as 'Category' in Supplementary Table 4A) in any of the gene structures is responsible for the gene expression patterns observed in APAs with KCNJ5 mutations. We observed significant relationships in upregulated and

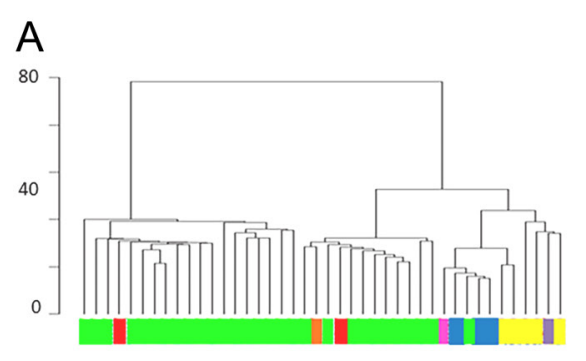

APA mutation KCNJ5

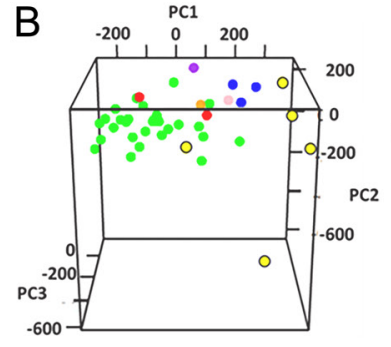

ATP2B3

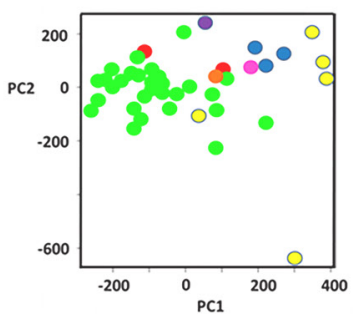

CTNNB1

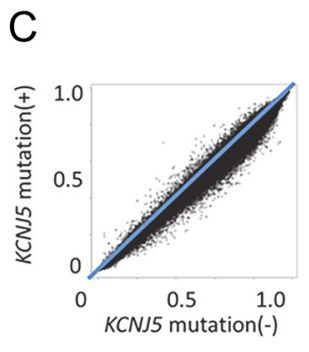

CPA

Figure 2

(A) Hierarchical clustering of the methylome profiles based on Euclidean distances in 37 APAs and 5CPAs. (B) Three-dimensional principal component analysis of the methylome profiles of 37 APAs. Component ratios used for the analysis are shown. Component ratios used for the analysis were PC1: $15.4 \%$, PC2: $9.2 \%$ and PC3: $6.7 \%$. (C) Scatter plotting of average $\beta$ values (all probes) of APAs with or without $K C N J 5$ mutations, indicating a global tendency for hypomethylation in APAs with KCNJ5 mutations. The blue line $(\mathrm{X}=\mathrm{Y})$ is displayed for reference. 


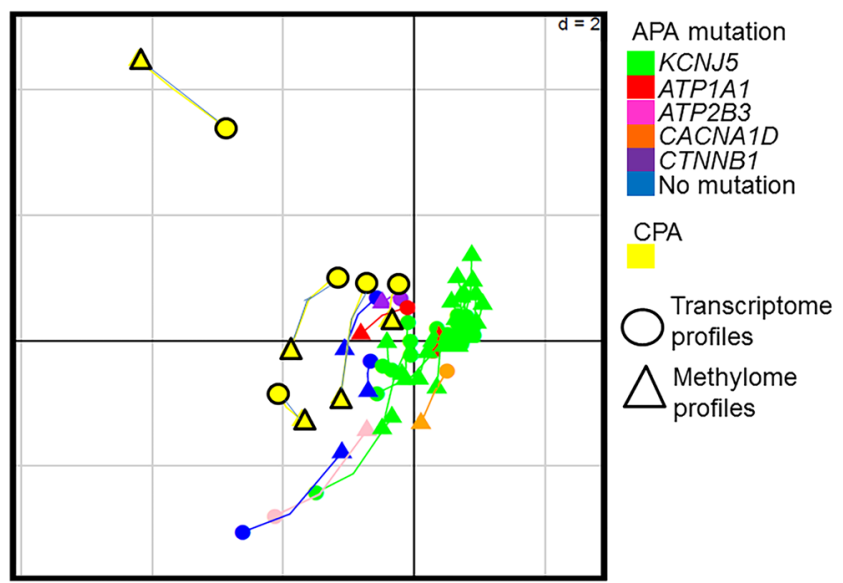

Figure 3

Multiple co-inertia analysis used as a dimension-reduction technique for integration of transcriptome and methylome analyses in 37 APAs and 5 CPAs. Circles and triangles represent transcriptome and methylome profiles, respectively.

hypomethylated genes at TSS200 and ExonBnd, and in downregulated and hypomethylated genes at ExonBnd and 3'UTR in APAs with KCNJ5 mutations (Supplementary Table 4A). The qualified genes that were extracted from these analyses are listed in Supplementary Table 4B.

\section{Pathway analysis}

GenMAPP pathway analysis was used to identify molecular characteristics of APAs with KCNJ5 mutations (Supplementary Table 5A and B). A transcriptomic comparison between APAs with KCNJ5 mutations and CPAs identified six upregulated gene pathways (by more than 1.5 fold) and 25 downregulated gene pathways (by less than 1.5 fold) in APAs with KCNJ5 mutations. Another transcriptomic comparison between APAs with and without KCNJ5 mutations identified 17 upregulated gene pathways (by more than 1.5 fold) and 29 downregulated gene pathways (by less than 1.5 fold) in APAs with KCNJ5 mutations. We integrated these two comparisons and extracted three upregulated gene pathways, such as the Wnt signaling pathway, and 11 downregulated gene pathways, such as the inflammatory response pathway, in APAs with KCNJ5 mutations when compared to CPAs and APAs without KCNJ5 mutations (Supplementary Table 5A and B).

\section{Transcriptomic comparison with normal adrenal cortex}

Transcriptome data from our 42 adrenal tumors were compared to microarray data from normal adrenal cortex (GEO accession no. GSE68889). Previous studies indicated morphological features of APAs with ATPase and CACNA1D mutation were similar to those of ZG and those of APAs with KCNJ5 mutation were similar to those of ZF (Monticone et al. 2015). To examine the relationship between mutation status and normal adrenal zonation in the point of molecular basis, we made 'KA' (KCNJ5 mutation) and 'AC' (ATP1A1, ATP2B3 and CACNA1D mutation) at first. For the rest APAs, CTNNB1 mutation and no mutation, were put into one group as ' $\mathrm{NC}$ ' because of their similar transcriptome profiles as shown in cluster analysis (Fig. 1A). Similarities in gene expression patterns between ZG and ZF and the 12 pair comparisons from the four adrenal tumor groups were evaluated using methods described in Supplementary Fig. 1 (Table 1). Highly significant consistence was observed in the KA vs $\mathrm{NC}$ (odds ratio $=6.24, \mathrm{FDR}=0.000$ ), KA vs CPA $(2.46$, $5.4 \mathrm{E}-10)$, AC vs NC $(5.38,0.000)$ and AC vs CPA $(2.69$, 5.8E-11) comparisons. Our analysis suggested similarities between APAs with KCNJ5, ATP1A1, ATP2B3 and CACNA1D mutations and ZG, and between APAs with CTNNB1 mutations, APA without any known mutations, and CPA and ZF. Based on our results, quantitative realtime PCR was performed to evaluate gene expressions of steroidogenic enzymes including CYP11B1, CYP11B2 and CYP17A1. CYP11B2 is known as a rate-limiting enzyme of aldosterone production. In contrast, CYP11B1 and CYP17A1 are considered as main pathway of cortisol production. Higher expression levels of CYP11B2 and lower expression levels of CYP17A1 were evident in APAs

Table 1 Transcriptome comparison between normal adrenal cortex and adrenal tumor using Fisher's exact test.

\begin{tabular}{|c|c|c|c|c|}
\hline $\begin{array}{l}\text { Adrenal } \\
\text { tumor pair }\end{array}$ & $\begin{array}{l}\text { Number of } \\
\text { differentiated } \\
\text { genes common } \\
\text { to ZG vs ZF }\end{array}$ & $\begin{array}{l}\text { Odds } \\
\text { ratio }\end{array}$ & $P$ value & FDR \\
\hline KA vs NC & 154 & 6.24 & $0.0 E+00$ & $0.0 E+00$ \\
\hline AC vs NC & 87 & 5.38 & $0.0 E+00$ & $0.0 E+00$ \\
\hline AC vs CPA & 74 & 2.69 & $9.5 E-13$ & $5.8 E-11$ \\
\hline KA vs CPA & 127 & 2.46 & $9.8 E-12$ & $5.4 E-10$ \\
\hline AC vs KA & 38 & 1.91 & $3.6 \mathrm{E}-04$ & $1.6 \mathrm{E}-02$ \\
\hline CPA vs NC & 20 & 1.75 & $1.7 \mathrm{E}-02$ & $6.5 E-01$ \\
\hline KA vs AC & 14 & 0.65 & $9.6 \mathrm{E}-01$ & $1.0 \mathrm{E}+00$ \\
\hline CPA vs KA & 35 & 0.55 & $1.0 \mathrm{E}+00$ & $1.0 \mathrm{E}+00$ \\
\hline CPA vs $A C$ & 11 & 0.33 & $1.0 \mathrm{E}+00$ & $1.0 \mathrm{E}+00$ \\
\hline NC vs CPA & 4 & 0.32 & $1.0 \mathrm{E}+00$ & $1.0 \mathrm{E}+00$ \\
\hline NC vs KA & 7 & 0.19 & $1.0 \mathrm{E}+00$ & $1.0 \mathrm{E}+00$ \\
\hline$N C$ vs $A C$ & 4 & 0.18 & $1.0 \mathrm{E}+00$ & $1.0 \mathrm{E}+00$ \\
\hline
\end{tabular}

In the comparison between $\mathrm{ZG}$ and $\mathrm{ZF}$, and in all paired comparison of adrenal tumors (KA: APAs with KCNJ5 mutation, NC: APAs with no mutations and CTNNB1 mutations, and AC: APAs with ATP1A1, ATP2B3, and CACNA1D mutations). Pairs with FDR $<0.001$ were indicated by bold.

Published by Bioscientifica Ltd 

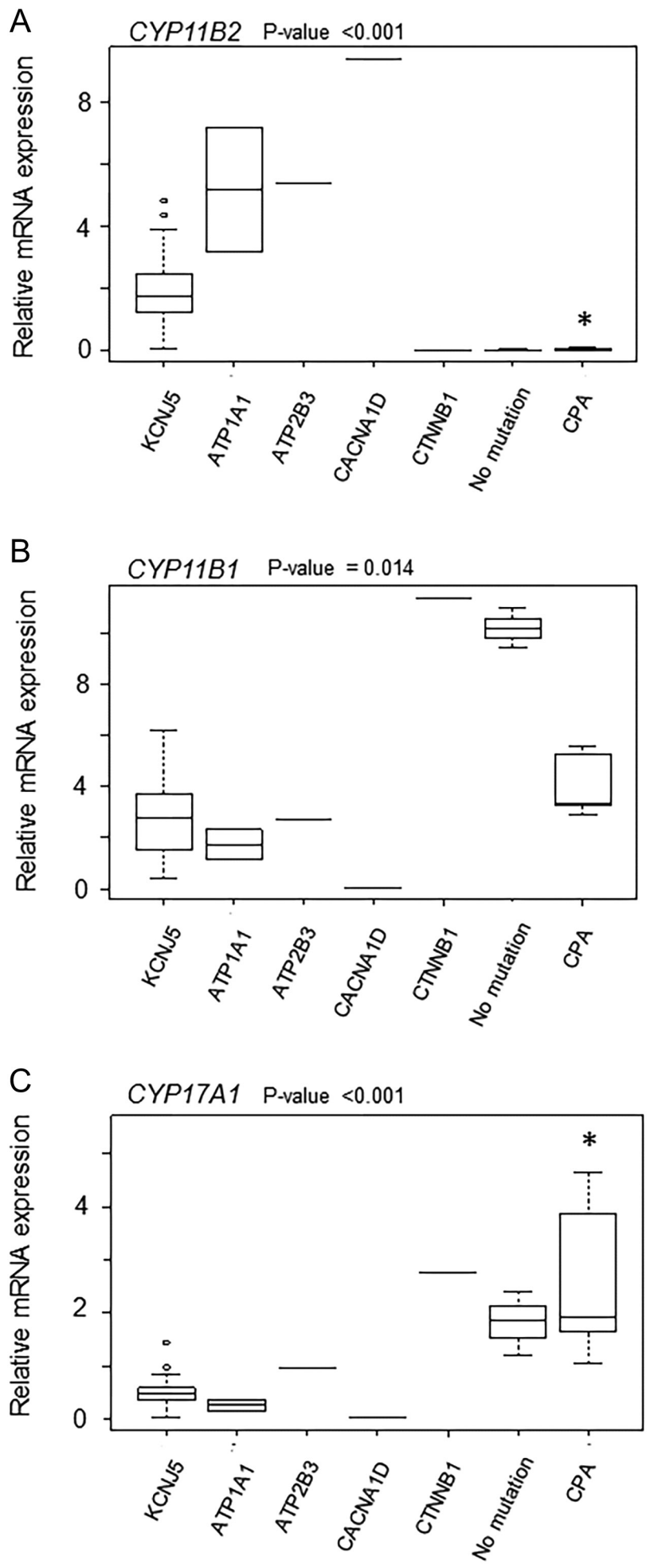

Figure 4

Comparison of mRNA levels of steroidogenic genes (CYP11B2 (A), CYP11B1 (B) and CYP17A1 (C)) among aldosterone-producing adenomas (APAs) with each mutational status and cortisol-producing adenoma (CPAs). Relative mRNA levels were calculated and presented as box plots. $P$ values were calculated using the Kruskal-Wallis rank sum test. *means $P<0.01$ compared to the levels of APAs with KCNJ5 mutation. with KCNJ5 mutation compared to CPA (Fig. 4A and C). Although it was difficult to find statistically significant relationships due to small numbers of samples, it seemed that CYP11B2 was highly expressed in APAs with ATP1A1, ATP2B3 CACNA1D mutation, and low expressed in APAs with CTNNB1 mutation and no mutation (Fig. 4A). In contrast, CYP11B1 and CYP17A1 were highly expressed in APAs with CTNNB1 mutation and no mutation and CPAs and low expressed in APAs with ATP1A1, ATP2B3, CACNA1D mutation (Fig. $4 \mathrm{~B}$ and C). Despite higher expression levels of CYP11B1 and CYP17A1, patients with CTNNB1 and those with no mutation did not meet the diagnostic criteria of autonomous cortisol secretion, except the one case with CTNNB1 mutation (serum cortisol after $1 \mathrm{mg}$ dexamethasone suppression test (DST) $>1.8 \mathrm{ng} / \mathrm{mL}$ ). The patient of APA with CTNNB1 mutation showed serum cortisol: $2.1 \mathrm{ng} / \mathrm{mL}$ after $1 \mathrm{mg}$ DST and $4.4 \mathrm{ng} / \mathrm{mL}$ at midnight, suggesting the possibility of weak autonomous cortisol secretion.

\section{Discussion}

We conducted comprehensive transcriptome and methylome analysis of 29 APAs with KCNJ5 mutations, 2 with ATP1A1 mutations, 1 with an ATP2B3 mutation, 1 with a CACNA1D mutation and 1 with a CTNNB1 mutation, along with 3 APAs with no known mutations. We used five CPAs as reference controls for adrenal tumors. In this study, we documented the molecular characteristics of APAs based on their mutational status. Here, we report the unique molecular characteristics of APAs with KCNJ5 mutations. The APAs with KCNJ5 mutations have global DNA hypomethylation and homogeneous transcriptomic profiles with changes in specific genes, such as those in the Wnt signaling, cytokine and inflammatory response pathways. In addition, comparisons with the GEO database indicated that the transcriptomic profile of APAs with KCNJ5 mutations is more similar to that of the ZG than that of the ZF. We performed double pairwise comparisons because direction (up or downregulation) should be considered to clarify similarities of transcriptome patterns between different tissues (Supplementary Fig. 1). Due to the different experimental systems used in the RNA sequence database for our adrenal tumors and the microarray-based GEO database for the normal adrenal cortex, we were unable to conduct direct comparisons between the two transcriptome databases.

Our transcriptome analysis of the 42 adrenal tumors indicated a heterogeneous distribution of transcriptomic profiles by principal component analysis. Although

Published by Bioscientifica Ltd. 
transcriptomic profiles of APAs with ATP1A1 and CACNA1D mutations were similar to those of APAs with KCNJ5 mutations, those of the APA with the CTNNB1 mutation and the APAs with no mutation were, notably, closer to those of CPAs. Methylome analysis of the 42 adrenal tumors also revealed a similar trend. APAs had heterogeneous methylomic profiles. Integration of transcriptome and methylome data using MCIA analysis also indicated the heterogeneity of APAs. Taken together, our findings indicate that although APAs fall into heterogeneous groups at the molecular level, there were similarities of APAs with KCNJ5 mutation to APAs with ATP1A1 and CACNA1D and closer relationship between APAs with CTNNB1 and no mutation and CPAs. Considering the types of mutations other than KCNJ5 are small in numbers, it is difficult to draw further conclusion. Nevertheless, it is conceivable that APAs with KCNJ5 mutation constitute one of the subgroups of APAs in the point of molecular characteristics, and our description may help to understand its feature.

Previous studies performed transcriptome analyses between APAs with and without KCNJ5 mutation (Boulkroun et al. 2012, Monticone et al. 2012). Boulkroun and coworkers showed no correlation with unique transcriptional profiles of APAs, which was inconsistent with our results (Boulkroun et al. 2012). To explain the discrepancy, it is important to mention that their constitution of somatic mutations other than KCNJ5mutated APAs was unknown. It was possible that their cohort included some APAs with ATP1A1 and CACNA1D mutation. Considering that transcriptome profiles of APAs with ATP1A1 and CACNA1D mutation were close to those of APAs with KCNJ5 mutation, and the potential proportion of APAs with ATP1A1 and CACNA1D mutation might affect their results. Monticone and coworkers also performed between APAs with and without KCNJ5 mutation and showed upregulated and downregulated genes (Monticone et al. 2012). It was of note that 15 of 20 most upregulated genes (ex. RELN, ALDH1A2, $P P P 2 R 2 B$ and et al.) and 10 of 20 last downregulated genes (ex. RASD2, SLC51A, GSTA1, and et al.) were also included in upregulated and downregulated genes in our comparison, respectively. Despite similarities between our and their analysis, there was a discrepancy about expression levels of CYP11B2. Monticone and coworkers showed upregulation of CYP11B2 in APAs with KCNJ5 mutation, which was not observed in our analysis. As shown in Fig. 4, expression levels of CYP11B2 were varied in mutation status and it was possible that their cohort might include higher proportion of APAs with CTNNB1 or no mutation, whose expression levels of CYP11B2 were lower than that of APAs with KCNJ5 mutation.

In contrast to previous studies showing that global DNA hypomethylation is a hallmark of APAs (Howard et al. 2014, Murakami et al. 2015b), the present study, which was based on differences in mutational status in APAs clearly revealed that global DNA hypomethylation status is a distinct characteristics in specific APAs with KCNJ5 mutations, rather than in all APAs. We found significant relationships between upregulated gene expression in genes such as TNNT2, GALNT9 and MPP4, and hypomethylation in their proximal promoter region (TSS200) in APAs with KCNJ5 mutations. Global DNA hypomethylation with specific gene upregulation appears to be a molecular hallmark of APAs with KCNJ5 mutations, although the pathophysiological role of each gene in APAs with KCNJ5 mutations remains undetermined.

Terms related to ion channels, transporters and the cell membrane were extracted using GO term analysis of the differentially expressed or methylated genes specific to APAs with KCNJ5 mutations. The known somatic mutations related to APA were mainly harbored in genes coding for ion channels and ATPases, whose dysfunctions are thought to induce the upregulation of $C Y P 11 B 2$, which is a rate-limiting enzyme in the aldosterone biosynthetic pathway (Zennaro et al. 2012). It is conceivable that the genes highlighted by the GO term analysis play important roles in the pathogenesis of APA. Furthermore, our GenMAPP pathway analysis for differentially expressed genes specific to APAs with KCNJ5 mutations revealed that the Wnt signaling pathway was upregulated in APAs with KCNJ5 mutations. Previous studies have suggested that the Wnt signaling pathway is involved in the development of adrenal tumors, including APAs (Zennaro et al. 2012). Our results therefore highlight the potential importance of the Wnt signaling pathway as a contributor to development of APAs with KCNJ5 mutations. In addition, 'cytokines and inflammatory response' and 'inflammatory response pathway' were downregulated in APAs with KCNJ5 mutations, as indicated by GenMAPP pathway analysis (Supplementary Table 5B). This is consistent with a previous study from our group indicated that inflammation-related genes are downregulated in APAs with KCNJ5 mutations (Murakami et al. 2016). Several previous studies have also suggested the existence of relationships between steroidogenesis and inflammationrelated genes in adrenal cells (Willenberg et al. 1998, Mikhaylova et al. 2007). Our findings therefore suggest the possible involvement of inflammation-related genes in the pathophysiology of APAs with KCNJ5 mutations.

Published by Bioscientifica Ltd 
Recent studies suggest a possible relationship between mutational status of APAs and their histopathology (Azizan et al. 2012a, Monticone et al. 2015). We therefore believed that comparisons between data obtained from the GEO database from normal adrenal cortical zonation (GSE68889) and the transcriptomes of our adrenocortical tumors would provide us with significant information regarding the pathogenesis of adrenocortical tumors. Our results indicated that APAs with KCNJ5, ATP1A1, $A T P 2 B 3$ and CACNA1D mutations are characterized by a ZG-like transcriptional pattern, whereas the APA with the CTNNB1 mutation, APAs without any known mutations and CPAs were characterized by ZF-like transcriptional patterns. Although previous histopathological studies have shown that APAs with KCNJ5 and ATP1A1 mutations consist of ZF-like cells and ZG-like cells, respectively (Azizan et al. 2012a, Monticone et al. 2015), other studies did not show same associations (Fernandes-Rosa et al. 2014). In particular, Boulkroun and coworkers showed that APA were composed of cells expressing ZG markers despite ZF-like cellular phenotype (Boulkroun et al. 2011), which were consistent with our findings. It is therefore conceivable that histological findings are not necessarily consistent with molecular profiles. In addition, it was of note that gene expression patterns of CYP11B1, CYP11B2 and CYP17A1 seemed to partly support our result. Previous study indicated that APAs with KCNJ5 mutation frequently expressed CYP11B1 and composed of ZF-like cells, and showed higher expression levels of CYP17A1 (Azizan et al. 2012a). However, the expression levels of CYP17A1 in APAs with KCNJ5 mutation were lower than those in APAs with no mutation in our analysis (Fig. 4B). This discrepancy may be partly attributable to the difference of patient profiles. It was possible that proportion of patients with autonomous cortisol secretion would affect the result. In addition, other mutations such as ATP1A1 and CACNA1D, which seemed to show lower expression levels of CYP17A1 in our analysis (Fig. 4C), could be included in their cohort. Higher expression levels of CYP11B2 and lower levels of CYP11B1 and CYP17A1 in APAs with KCNJ5 mutation compared to APAs with CTNNB1 and no mutation seemed to be consistent with characteristics of ZG cells rather than ZF cells, which was indicated in our transcriptome comparison (Table 1). In addition, low expression of CYP11B2 and high expression of CYP11B1 and CYP17A1 may be common in APAs with CTNNB1 and no mutation and CPAs. High expression of CYP11B2 and low expression of CYP11B1 and CYP17A1 may be common in APAs with ATP1A1, ATP2B3 and CACNA1D. Although characteristics of APAs with KCNJ5 mutation were rather unclear in Fig. 4, there was a tendency suggesting that APAs with ATP1A1, ATP2B3 and CACNA1D constitute a group from ZG cells, and APAs with CTNNB1 and no mutation and CPAs constitute a group from ZF cells. Further analysis of the relationships between histology and molecular characteristics in APAs are required to clarify whether the heterogeneity in APAs is attributable to differences in cell origin. Nevertheless, a ZG-like transcriptional pattern and a ZF-like morphology appear to be distinct characteristics of APAs with KCNJ5 mutations, which form a unique subgroup among the APAs. Considering, $\mathrm{Wu}$ and coworkers suggested that CTNNB1 mutations might be more related to tumorigenesis rather than excessive aldosterone production (Wu et al. 2017), similarities between APAs with CTNNB1 mutation and CPAs observed in this study will provide new insights into molecular mechanisms of adrenal tumorigenesis. It is to be elucidated whether CTNNB1 mutation is related to cortisol excessive production.

There are some limitations to this study. Except for APAs with KCNJ5 mutation, the numbers of adrenal tumor were small in our analysis due to its rarity as clinical samples. It was necessary to keep in mind that clinical characteristics such as sex or age, which are significantly associated with KCNJ5 mutation status, could affect our results. A direct comparison of molecular characteristics, including those of the transcriptome and methylome, between nonfunctional adrenal adenomas and normal adrenal cells would have been a more preferable reference control in this study. However, there is little opportunity to obtain sufficient numbers of non-functional adrenal adenomas and normal adrenal cortex samples. In addition, we were unable to perform the methylomic comparison between normal adrenal cortex and our adrenal tumors, since the suitable dataset was not available in the public database and it is technically difficult to perform methylome analysis using a small amount of sample, such as that lasercaptured from each adrenocortical zonation. Finally, due to the small sample number, detailed integration of the transcriptome, methylome and pathway analyses could not be performed for each individual APA mutational status, other than that for the KCNJ5 mutation.

This study revealed the molecular heterogeneity of APAs. APAs with somatic KCNJ5 mutations constitute a subgroup of APAs, as they have global DNA hypomethylation and transcriptomic profiles accompanied by changes in specific genes, such as those in the Wnt signaling, cytokine and inflammatory response pathways. In addition, comparisons with data in the GEO database indicated that the transcriptomic

Published by Bioscientifica Ltd 
profiles of APAs with KCNJ5 mutations are more similar to those of ZG than those of ZF. In addition, the coexistence of the ZG-like transcriptional pattern shown in this study and the previously reported ZF-like morphology are considered to be hallmarks of APAs with KCNJ5 mutations. It is conceivable that the heterogeneity of APAs is attributed to differences in cell origins. The molecular characterization of APAs via our integration of transcriptome and methylome analyses provides new insights into the pathogenic mechanism(s) of APAs.

Supplementary data

This is linked to the online version of the paper at http://dx.doi.org/10.1530/ ERC-17-0117.

\section{Declaration of interest}

The authors declare that there is no conflict of interest that could be perceived as prejudicing the impartiality of the research reported.

\section{Funding}

This study was conducted as a part of the Japan Primary Aldosteronism Study (JPAS) supported by a Research Grant from the Japan Agency for Medical Research and Development (AMED) (no. 15Aek0109122) and partially supported by Grants-in-Aid for Scientific Research from the Ministry of Education, Science, Sports and Culture of Japan (T Y, 16K08962; and $\mathrm{M} \mathrm{M}, 16 \mathrm{~K} 19392)$

\section{Acknowledgements}

The authors thank all other staff members of the Department of Molecular Endocrinology and Metabolism, Tokyo Medical and Dental University and DNA Chip Research, Inc.

\section{References}

Akerstrom T, Crona J, Verdugo AD, Starker LF, Cupisti K, Willenberg HS, Knoefel WT, Saeger W, Feller A, Ip J, et al. 2012 Comprehensive re-sequencing of adrenal aldosterone producing lesions reveal three somatic mutations near the KCNJ5 potassium channel selectivity filter. PLOS ONE 7 e41926. (doi:10.1371/journal.pone.0041926)

Akerstrom T, Maharjan R, Sven Willenberg H, Cupisti K, Ip J, Moser A, Stalberg P, Robinson B, Alexander Iwen K, Dralle H, et al. 2016 Activating mutations in CTNNB1 in aldosterone producing adenomas. Scientific Reports 6 19546. (doi:10.1038/srep19546)

Akerstrom T, Willenberg HS, Cupisti K, Ip J, Backman S, Moser A, Maharjan R, Robinson B, Iwen KA, Dralle H, et al. 2015 Novel somatic mutations and distinct molecular signature in aldosteroneproducing adenomas. Endocrine-Related Cancer 22 735-744. (doi:10.1530/ERC-15-0321)

Arnesen T, Glomnes N, Stromsoy S, Knappskog S, Heie A, Akslen LA Grytaas M, Varhaug JE, Gimm O \& Brauckhoff M 2013 Outcome after surgery for primary hyperaldosteronism may depend on KCNJ5 tumor mutation status: a population-based study from Western Norway. Langenbecks Archives of Surgery 398 869-874. (doi:10.1007/ s00423-013-1093-2)
Azizan EA, Lam BY, Newhouse SJ, Zhou J, Kuc RE, Clarke J, Happerfield L, Marker A, Hoffman GJ \& Brown MJ 2012a Microarray, qPCR, and KCNJ5 sequencing of aldosterone-producing adenomas reveal differences in genotype and phenotype between zona glomerulosaand zona fasciculata-like tumors. Journal of Clinical Endocrinology and Metabolism 97 E819-E829. (doi:10.1210/jc.2011-2965)

Azizan EA, Murthy M, Stowasser M, Gordon R, Kowalski B, Xu S, Brown MJ \& O'Shaughnessy KM 2012b Somatic mutations affecting the selectivity filter of KCNJ5 are frequent in 2 large unselected collections of adrenal aldosteronomas. Hypertension 59 587-591. (doi:10.1161/HYPERTENSIONAHA.111.186239)

Azizan EA, Poulsen H, Tuluc P, Zhou J, Clausen MV, Lieb A, Maniero C, Garg S, Bochukova EG, Zhao W, et al. 2013 Somatic mutations in ATP1A1 and CACNA1D underlie a common subtype of adrenal hypertension. Nature Genetics 45 1055-1060. (doi:10.1038/ng.2716)

Beuschlein F, Boulkroun S, Osswald A, Wieland T, Nielsen HN, Lichtenauer UD, Penton D, Schack VR, Amar L, Fischer E, et al. 2013 Somatic mutations in ATP1A1 and ATP2B3 lead to aldosteroneproducing adenomas and secondary hypertension. Nature Genetics 45 440-444. (doi:10.1038/ng.2550)

Boulkroun S, Samson-Couterie B, Golib-Dzib JF, Amar L, Plouin PF, Sibony M, Lefebvre $\mathrm{H}$, Louiset E, Jeunemaitre X, Meatchi T, et al. 2011 Aldosterone-producing adenoma formation in the adrenal cortex involves expression of stem/progenitor cell markers. Endocrinology 152 4753-4763. (doi:10.1210/en.2011-1205)

Boulkroun S, Beuschlein F, Rossi GP, Golib-Dzib JF, Fischer E, Amar L, Mulatero P, Samson-Couterie B, Hahner S, Quinkler M, et al. 2012 Prevalence, clinical, and molecular correlates of KCNJ5 mutations in primary aldosteronism. Hypertension 59 592-598. (doi:10.1161/ HYPERTENSIONAHA.111.186478)

Choi M, Scholl UI, Yue P, Bjorklund P, Zhao B, Nelson-Williams C, Ji W, Cho Y, Patel A, Men CJ, et al. $2011 \mathrm{~K}+$ channel mutations in adrenal aldosterone-producing adenomas and hereditary hypertension. Science 331 768-772. (doi:10.1126/science.1198785)

Daniil G, Fernandes-Rosa FL, Chemin J, Blesneac I, Beltrand J, Polak M, Jeunemaitre X, Boulkroun S, Amar L, Strom TM, et al. 2016 CACNA1H mutations are associated with different forms of primary aldosteronism. EBioMedicine 13 225-236. (doi:10.1016/j. ebiom.2016.10.002)

Das PM \& Singal R 2004 DNA methylation and cancer. Journal of Clinical Oncology 22 4632-4642. (doi:10.1200/JCO.2004.07.151)

Dennis G Jr, Sherman BT, Hosack DA, Yang J, Gao W, Lane HC \& Lempicki RA 2003 DAVID: database for annotation, visualization, and integrated discovery. Genome Biology 4 R60. (doi:10.1186/ gb-2003-4-9-r60)

Fassnacht M, Arlt W, Bancos I, Dralle H, Newell-Price J, Sahdev A, Tabarin A, Terzolo M, Tsagarakis S \& Dekkers OM 2016 Management of adrenal incidentalomas: European society of endocrinology clinical practice guideline in collaboration with the european network for the study of adrenal tumors. European Journal of Endocrinology 175 G1-G34. (doi:10.1530/EJE-16-0467)

Fernandes-Rosa FL, Williams TA, Riester A, Steichen O, Beuschlein F, Boulkroun S, Strom TM, Monticone S, Amar L, Meatchi T, et al. 2014 Genetic spectrum and clinical correlates of somatic mutations in aldosterone-producing adenoma. Hypertension 64 354-361. (doi:10.1161/HYPERTENSIONAHA.114.03419)

Hannemann A \& Wallaschofski H 2012 Prevalence of primary aldosteronism in patient's cohorts and in population-based studies - a review of the current literature. Hormone and Metabolic Research 44 157-162. (doi:10.1055/s-0031-1295438)

Howard B, Wang Y, Xekouki P, Faucz FR, Jain M, Zhang L, Meltzer PG, Stratakis CA \& Kebebew E 2014 Integrated analysis of genome-wide methylation and gene expression shows epigenetic regulation of CYP11B2 in aldosteronomas. Journal of Clinical Endocrinology and Metabolism 99 E536-E543. (doi:10.1210/ jc.2013-3495) 
Jaenisch R \& Bird A 2003 Epigenetic regulation of gene expression: how the genome integrates intrinsic and environmental signals. Nature Genetics 33 245-254. (doi:10.1038/ng1089)

Kitamoto T, Suematsu S, Matsuzawa Y, Saito J, Omura M \& Nishikawa T 2015 Comparison of cardiovascular complications in patients with and without KCNJ5 gene mutations harboring aldosteroneproducing adenomas. Journal of Atherosclerosis and Thrombosis 22 191-200. (doi:10.5551/jat.24455)

Lenzini L, Rossitto G, Maiolino G, Letizia C, Funder JW \& Rossi GP 2015 A meta-analysis of somatic KCNJ5 K(+) channel mutations in 1636 patients with an aldosterone-producing adenoma. Journal of Clinical Endocrinology and Metabolism 100 E1089-E1095. (doi:10.1210/jc.2015-2149)

Meng C, Kuster B, Culhane AC \& Gholami AM 2014 A multivariate approach to the integration of multi-omics datasets. $B M C$ Bioinformatics 15 162. (doi:10.1186/1471-2105-15-162)

Mikhaylova IV, Kuulasmaa T, Jaaskelainen J \& Voutilainen R 2007 Tumor necrosis factor-alpha regulates steroidogenesis, apoptosis, and cell viability in the human adrenocortical cell line NCI-H295R. Endocrinology 148 386-392. (doi:10.1210/en.2006-0726)

Milliez P, Girerd X, Plouin PF, Blacher J, Safar ME \& Mourad JJ 2005 Evidence for an increased rate of cardiovascular events in patients with primary aldosteronism. Journal of the American College of Cardiology 45 1243-1248. (doi:10.1016/j.jacc.2005.01.015)

Monticone S, Hattangady NG, Nishimoto K, Mantero F, Rubin B, Cicala MV, Pezzani R, Auchus RJ, Ghayee HK, Shibata H, et al. 2012 Effect of KCNJ5 mutations on gene expression in aldosterone-producing adenomas and adrenocortical cells. Journal of Clinical Endocrinology and Metabolism 97 E1567-E1572. (doi:10.1210/jc.2011-3132)

Monticone S, Castellano I, Versace K, Lucatello B, Veglio F, GomezSanchez CE, Williams TA \& Mulatero P 2015 Immunohistochemical, genetic and clinical characterization of sporadic aldosteroneproducing adenomas. Molecular and Cellular Endocrinology $\mathbf{4 1 1}$ 146-154. (doi:10.1016/j.mce.2015.04.022)

Murakami M, Yoshimoto T, Minami I, Bouchi R, Tsuchiya K, Hashimoto K, Izumiyama H, Fujii Y, Endo T, Akashi T, et al. 2015a A novel somatic deletion mutation of ATP2B3 in aldosterone-producing adenoma. Endocrine Pathology 26 328-333. (doi:10.1007/s12022-015-9400-9)

Murakami M, Yoshimoto T, Nakabayashi K, Tsuchiya K, Minami I, Bouchi $\mathrm{R}$, Izumiyama H, Fujii Y, Abe K, Tayama C, et al. 2015b Integration of transcriptome and methylome analysis of aldosterone-producing adenomas. European Journal of Endocrinology 173 185-195. (doi:10.1530/EJE-15-0148)

Murakami M, Yoshimoto T, Nakano Y, Tsuchiya K, Minami I, Bouchi R, Fujii Y, Nakabayashi K, Hashimoto K, Hata K, et al. 2016 Expression of inflammation-related genes in aldosterone-producing adenomas with KCNJ5 mutation. Biochemical and Biophysical Research Communications 476 614-619. (doi:10.1016/j.bbrc.2016.06.007)

Nishikawa T, Omura M, Satoh F, Shibata H, Takahashi K, Tamura N, Tanabe A \& Task Force Committee on Primary Aldosteronism TJES 2011 Guidelines for the diagnosis and treatment of primary aldosteronism - the Japan Endocrine Society 2009. Endocrine Journal 58 711-721. (doi:10.1507/endocrj.EJ11-0133)

Nishimoto K, Tomlins SA, Kuick R, Cani AK, Giordano TJ, Hovelson DH, Liu CJ, Sanjanwala AR, Edwards MA, Gomez-Sanchez CE, et al. 2015 Aldosterone-stimulating somatic gene mutations are common in normal adrenal glands. PNAS 112 E4591-E4599. (doi:10.1073/ pnas.1505529112)
Osswald A, Fischer E, Degenhart C, Quinkler M, Bidlingmaier M, Pallauf A, Lang K, Mussack T, Hallfeldt K, Beuschlein F, et al. 2013 Lack of influence of somatic mutations on steroid gradients during adrenal vein sampling in aldosterone-producing adenoma patients. European Journal of Endocrinology 169 657-663. (doi:10.1530/EJE-13-0551)

Plouin PF, Amar L \& Chatellier G 2004 Trends in the prevalence of primary aldosteronism, aldosterone-producing adenomas, and surgically correctable aldosterone-dependent hypertension. Nephrology, Dialysis, Transplantation 19 774-777. (doi:10.1093/ndt/ gfh112)

Rossi GP, Cesari M, Letizia C, Seccia TM, Cicala MV, Zinnamosca L, Kuppusamy M, Mareso S, Sciomer S, Iacobone M, et al. 2014 KCNJ5 gene somatic mutations affect cardiac remodelling but do not preclude cure of high blood pressure and regression of left ventricular hypertrophy in primary aldosteronism. Journal of Hypertension 32 1514-1522. (doi:10.1097/HJH.0000000000000186)

Scholl UI, Healy JM, Thiel A, Fonseca AL, Brown TC, Kunstman JW, Horne MJ, Dietrich D, Riemer J, Kucukkoylu S, et al. 2015a Novel somatic mutations in primary hyperaldosteronism are related to the clinical, radiological and pathological phenotype. Clinical Endocrinology 83 779-789. (doi:10.1111/cen.12873)

Scholl UI, Stolting G, Nelson-Williams C, Vichot AA, Choi M, Loring E, Prasad ML, Goh G, Carling T, Juhlin CC, et al. 2015b Recurrent gain of function mutation in calcium channel CACNA1H causes early-onset hypertension with primary aldosteronism. eLife $\mathbf{4}$ e06315. (doi:10.7554/eLife.06315)

Taguchi R, Yamada M, Nakajima Y, Satoh T, Hashimoto K, Shibusawa N, Ozawa A, Okada S, Rokutanda N, Takata D, et al. 2012 Expression and mutations of KCNJ5 mRNA in Japanese patients with aldosterone-producing adenomas. Journal of Clinical Endocrinology and Metabolism 97 1311-1319. (doi:10.1210/jc.2011-2885)

Wang B, Li X, Zhang X, Ma X, Chen L, Zhang Y, Lyu X, Tang Y, Huang Q, Gao Y, et al. 2015 Prevalence and characterization of somatic mutations in Chinese aldosterone-producing adenoma patients. Medicine 94 e708. (doi:10.1097/MD.0000000000000708)

Willenberg HS, Stratakis CA, Marx C, Ehrhart-Bornstein M, Chrousos GP \& Bornstein SR 1998 Aberrant interleukin-1 receptors in a cortisolsecreting adrenal adenoma causing Cushing's syndrome. New England Journal of Medicine 339 27-31. (doi:10.1056/ NEJM199807023390105)

Williams TA, Monticone S, Schack VR, Stindl J, Burrello J, Buffolo F, Annaratone L, Castellano I, Beuschlein F, Reincke M, et al. 2014 Somatic ATP1A1, ATP2B3, and KCNJ5 mutations in aldosteroneproducing adenomas. Hypertension 63 188-195. (doi:10.1161/ HYPERTENSIONAHA.113.01733)

Williams TA, Peitzsch M, Dietz AS, Dekkers T, Bidlingmaier M, Riester A, Treitl M, Rhayem Y, Beuschlein F, Lenders JW, et al. 2016 Genotypespecific steroid profiles associated with aldosterone-producing adenomas. Hypertension 67 139-145. (doi:10.1161/ HYPERTENSIONAHA.115.06186)

Wu VC, Wang SM, Chueh SJ, Yang SY, Huang KH, Lin YH, Wang JJ, Connolly R, Hu YH, Gomez-Sanchez CE, et al. 2017 The prevalence of CTNNB1 mutations in primary aldosteronism and consequences for clinical outcomes. Scientific Reports 7 39121. (doi:10.1038/ srep39121)

Zennaro MC, Jeunemaitre X \& Boulkroun S 2012 Integrating genetics and genomics in primary aldosteronism. Hypertension $60580-588$. (doi:10.1161/HYPERTENSIONAHA.111.188250)

Received in final form 21 June 2017

Accepted 26 July 2017

Accepted Preprint published online 26 July 2017 http://erc.endocrinology-journals.org DOI: 10.1530/ERC-17-0117
C 2017 Society for Endocrinology Printed in Great Britain
Published by Bioscientifica Ltd 